IJMMS 2003:7, 397-403

PII. S0161171203107090

http://ijmms.hindawi.com

(c) Hindawi Publishing Corp.

\title{
HILBERT SERIES AND APPLICATIONS TO GRADED RINGS
}

\author{
SELMA ALTINOK
}

Received 23 July 2001 and in revised form 14 March 2002

\begin{abstract}
This paper contains a number of practical remarks on Hilbert series that we expect to be useful in various contexts. We use the fractional Riemann-Roch formula of Fletcher and Reid to write out explicit formulas for the Hilbert series $P(t)$ in a number of cases of interest for singular surfaces (see Lemma 2.1) and 3-folds. If $X$ is a $\mathbb{Q}$-Fano 3 -fold and $S \in\left|-K_{X}\right|$ a $K 3$ surface in its anticanonical system (or the general elephant of $X$ ), polarised with $D=O_{S}\left(-K_{X}\right)$, we determine the relation between $P_{X}(t)$ and $P_{S, D}(t)$. We discuss the denominator $\prod\left(1-t^{a_{i}}\right)$ of $P(t)$ and, in particular, the question of how to choose a reasonably small denominator. This idea has applications to finding $K 3$ surfaces and Fano 3-folds whose corresponding graded rings have small codimension. Most of the information about the anticanonical ring of a Fano 3 -fold or $K 3$ surface is contained in its Hilbert series. We believe that, by using information on Hilbert series, the classification of $\mathbb{Q}$-Fano 3-folds is too close. Finding $K 3$ surfaces are important because they occur as the general elephant of a $\mathbb{Q}$-Fano 3-fold.
\end{abstract}

2000 Mathematics Subject Classification: 14Q10, 14Q15, 32S25, 13A02.

1. Introduction. We work with graded rings $R=\bigoplus_{n \geq 0} R_{n}$ that are finitely generated over an algebraically closed field $k$ of characteristic 0 and satisfy $R_{0}=k$. The Hilbert function of $R$ is the numerical function $P_{n}=\operatorname{dim} R_{n}$ for $n \geq 0$; the Hilbert series $P(t)$ or $P_{R}(t)$ of $R$ is the formal power series defined by $P(t)=\sum P_{n} t^{n}$. It is elementary and well known that $P(t)$ is a rational function of $t$. In fact, if $x_{1}, \ldots, x_{d}$ are homogeneous elements of weight wt $x_{i}=a_{i}$ generating $R$ (or more generally, generating a subring over which $R$ is finite), then $\prod\left(1-t^{a_{i}}\right) P(t)=Q(t)$ is a polynomial.

\section{Fractional Riemann-Roch formula}

2.1. Surfaces with Du Val singularities. We use the definitions and notation of Reid [9] for singularities. If $S$ is a projective surface with Du Val singularities and $D$ a Weil divisor on $S$, then some multiple $r D$ is Cartier, and there is a formula [9, Theorem 9.1]

$$
\chi\left(S, \mathscr{O}_{S}(D)\right)=\chi\left(\mathscr{O}_{S}\right)+\frac{1}{2}\left(D^{2}-D K_{S}\right)+\sum_{P} c_{P}(D),
$$

where $c_{P}(D) \in \mathbb{Q}$ is a fractional contribution due to the singularity of $S$ and 
$\mathrm{O}_{S}(D)$ at $P$. Here, $D K_{S} \in \mathbb{Z}$ is the intersection number with the canonical class, and $D^{2} \in \mathbb{Q}$ the self-intersection of the $\mathbb{Q}$-Cartier divisor $D$. Moreover, $c_{P}(D)$ can be written as a sum

$$
c_{P}(D)=-\sum_{(r, a) \in \mathscr{B}} \frac{a(r-a)}{2 r}
$$

over a basket $\mathscr{B}=\{(r, a)\}$ with $0<a<r$ and each $a$ is coprime to $r$. Here, the basket appears simply as a list of combinatorial data for computing the right-hand side of (2.2); Reid [9, Section 9] interprets (2.1) and (2.2) as the singularity of $S$, and $O_{S}(D)$ at $P$ has the same effect on $\chi\left(S, \mathcal{O}_{S}(D)\right)$ as a basket $\mathscr{B}$ of virtual cyclic quotient singularities of type $(1 / r)(1,-1)$, at which $\mathbb{O}_{S}(D)$ is locally isomorphic to the eigensheaf of $\varepsilon^{a}$ (see [9, Chapter III] for definition).

It follows from this interpretation and the proof of [9, Theorem 9.1] that for $n \in \mathbb{Z}$

$$
c_{P}(n D)=-\sum_{(r, a) \in \mathscr{B}} \frac{\overline{n a}(r-\overline{n a})}{2 r},
$$

where, for each $(r, a) \in \mathscr{B}$, the bar stands for the smallest positive residue modulo $r$.

LEMMA 2.1. Let $S$ be a surface with Du Val singularities and $D$ a Weil divisor. We assume that $H^{i}\left(S, O_{S}(n D)\right)=0$ for all $i>0$ and for all $n \geq 1$. Then, the graded ring $R(S, D)=\bigoplus_{n \geq 0} H^{0}\left(S, O_{S}(n D)\right)$ has Hilbert series

$$
\begin{aligned}
P_{S}(t)= & \frac{1+(\chi-1) t}{1-t}+\frac{t+t^{2}}{2(1-t)^{3}} D^{2}-\frac{t}{2(1-t)^{2}} D K_{S} \\
& -\sum_{(r, a) \in \mathscr{B}} \frac{\sum_{n=1}^{r-1} \overline{a n}(r-\overline{a n}) t^{n}}{2 r\left(1-t^{r}\right)} .
\end{aligned}
$$

Here, $x=x\left(\mathbb{O}_{S}\right)$.

Proof. The first three terms of (2.4) expand out as

$$
\begin{gathered}
1+\chi t+\chi t^{2}+\cdots, \quad \frac{\left(t+2^{2} t^{2}+3^{2} t^{3}+\cdots\right) D^{2}}{2}, \\
-\frac{\left(t+2 t^{2}+3 t^{2}+\cdots\right) D K_{S}}{2}
\end{gathered}
$$

respectively, corresponding to the sum over $n D$ of first three terms of (2.1). For each element of the basket, the denominator $1-t^{r}$ has the effect of repeating the contribution $-\overline{n a}(r-\overline{n a}) / 2 r$ from (2.3) periodically over the intervals $[0, r],[r, 2 r], \ldots$ (zero at the endpoints), giving the last term of (2.1).

2.2. 3-folds with canonical singularities. Let $X$ be a projective 3-fold with canonical singularities and $A$ a Weil divisor. To use the formulas of [9, Section 10], assume that, at every singular point $P \in X$, we have $O_{X}(A) \cong 0_{X}\left(l K_{X}\right)$ for 
some $l$ (possibly depending on $P$ ). Then, [9, Theorem 10.2] states that

$$
\chi(X, A)=\chi\left(O_{X}\right)+\frac{1}{6} A^{3}-\frac{1}{4} A^{2} K_{X}+\frac{1}{12} A\left(K_{X}^{2}+c_{2}\right)+\sum c_{Q}(A),
$$

where $c_{Q}(A)$ is a sum $\sum_{\mathscr{B}} c(r, a, l)$ taken over a basket $\mathscr{B}=\{(r, a, l)\}$, with $a$ and $l$ coprime to $r$, and the contributions are

$$
\begin{aligned}
c(r, a, l) & =-\frac{r^{2}-1}{12 r} l+\sum_{i=1}^{l-1} \frac{\overline{a i}(r-\overline{a i})}{2 r} \\
& =\frac{r^{2}-1}{12 r}(r-l)-\sum_{i=l}^{r-1} \frac{\overline{a i}(r-\overline{a i})}{2 r} .
\end{aligned}
$$

The interpretation here is that $c(r, a, l)$ is the contribution from a singularity of type $(1 / r)(a, 1,-1)$ at which $A$ is locally the $\varepsilon^{a l}$ eigensheaf.

We can write out the other terms in the Hilbert series by analogy with Lemma 2.1 (see the following section). The contribution made by each element $(r, a, l) \in \mathscr{B}$ to the Hilbert series is thus

$$
\sum_{n=1}^{\infty} c(r, a, n l) t^{n}=\frac{1}{1-t^{r}} \sum_{n=1}^{r-1} c(r, a, n l) t^{n} .
$$

REMARK 2.2. The two formulas (2.7) and (2.8) are equal because

$$
\sum_{i=1}^{r-1} \overline{a i}(r-\overline{a i})=\sum_{i=1}^{r-1} i(r-i)=\frac{1}{6} r\left(r^{2}-1\right) ;
$$

moreover, they also hold for $l$ not in $[0, r]$ (the expression only depends on $l$ modulo $r$ ).

In dealing with a single divisor $A$, we may assume that $l$ is coprime to $r$; but the proof of [9, Section 10] is valid for any $l$. Thus, we can use formulas (2.7) and (2.8) for the contribution $c(r, a, n l)$ for any $n$ in calculating $\chi(n A)$.

\section{Fano 3-folds and $K 3$ surfaces}

3.1. Fano 3-folds. Let $X$ be a Fano 3-fold, that is, a 3-fold with canonical singularities and ample anticanonical class $A=-K_{X}$. Standard use of vanishing gives

$$
P_{n}=h^{0}(X, n A)=\chi(O(n A)), \text { for } n \geq 0 .
$$

Now, $\mathcal{X}\left(\mathscr{O}_{X}\right)=1$ and the basket of $X$ is $\mathscr{B}=\{(r, a,-1)\}$, and by [9, Corollary 10.3], we have

$$
\frac{1}{12} A c_{2}=-\frac{1}{12} K_{X} c_{2}=2-\sum_{\mathscr{B}} \frac{r^{2}-1}{12 r} .
$$


Using all this, formulas (2.6) and (2.7) specialise to give

$$
P_{n}=2 n+1+\frac{1}{12} n(n+1)(2 n+1) A^{3}+\sum_{\mathscr{B}}\left(-\frac{r^{2}-1}{12 r} n+c(r, a,-n)\right) .
$$

Now, consider $P_{n}-P_{n-1}$. To handle the second term, we use

$$
n(n+1)(2 n+1)-(n-1)(n)(2 n-1)=6 n^{2} .
$$

By (2.8), the bracketed expression inside the sum over $\mathscr{B}$ equals

$$
-\sum_{i=-n}^{r-1} \frac{\overline{a i}(r-\overline{a i})}{2 r}
$$

and the difference from $n$ to $n-1$ is just one term of the sum. We obtain

$$
P_{n}-P_{n-1}=2+\frac{1}{2} n^{2} A^{3}-\sum_{(r, a,-1) \in \mathscr{B}} \frac{\overline{a n}(r-\overline{a n})}{2 r} .
$$

Since $P_{n}-P_{n-1}$ is the coefficient of $t^{n}$ in $(1-t) P_{X}(t)$, arguing as in Lemma 2.1 gives the following result.

COROLLARY 3.1. The Hilbert series of a Fano 3-fold $X$ is

$$
P_{X}(t)=\frac{1+t}{(1-t)^{2}}+\frac{t(1+t)}{2(1-t)^{4}} A^{3}-\sum_{(r, a,-1) \in \mathscr{B}} \frac{\sum_{n=1}^{r-1} \overline{a n}(r-\overline{a n}) t^{n}}{2 r(1-t)\left(1-t^{r}\right)} .
$$

3.2. $K 3$ surfaces. For a $K 3$ surface $S$ with Du Val singularities and a Weil divisor $D$, Lemma 2.1 specialises to give the Hilbert series of $P_{n}=h^{0}(S, n D)$ in the form

$$
P_{S}(t)=\frac{1+t}{1-t}+\frac{t(1+t)}{2(1-t)^{3}} D^{2}-\sum_{(r, a) \in \mathscr{B}} \frac{\sum_{n=1}^{r-1} \overline{a n}(r-\overline{a n}) t^{n}}{2 r\left(1-t^{r}\right)}
$$

COROLlary 3.2. If $X$ is a Fano 3-fold polarised by $A=-K_{X}$ and $S \in\left|-K_{X}\right|$ a K3 surface polarised by $D=A_{\mid S}$, then

$$
P_{S}(t)=(1-t) P_{X}(t)
$$

This result follows, of course, from the restriction exact sequence

$$
0 \longrightarrow \mathrm{O}_{X}((n-1) A) \longrightarrow \mathrm{O}_{X}(n A) \longrightarrow \mathrm{O}_{S}(n D) \longrightarrow 0
$$

The point, however, is that the corollary gives a formula for the Hilbert series $P_{X}(t)$ of the Fano 3 -fold in terms of simpler data for a $K 3$ surface. This formula is valid even if there is no $K 3$ surface $S \in\left|-K_{X}\right|$, for example, if $P_{1}(X)=0$ so that $\left|-K_{X}\right|=\varnothing$. Compare Corti, Pukhlikov, and Reid [5, Remark 7.2.3]. 
4. Applications. In [2], we studied polarised $K 3$ surfaces $S$ in terms of their graded rings $R(S, D)$. We also studied Fano 3-folds $X$ in a similar way. The first step in our strategy consisted of finding suitable weights $a_{0}, a_{1}$, and $a_{2}$ so that

$$
\left(1-t^{a_{0}}\right)\left(1-t^{a_{1}}\right)\left(1-t^{a_{2}}\right) P(t)
$$

is a polynomial with positive coefficients and $a_{0}, a_{1}$, and $a_{2}$ are "fairly small." This is a combinatorial analogue of finding a polynomial subring $k\left[x_{0}, x_{1}, x_{2}\right] \subset$ $R(S, D)$ over which $R(S, D)$ is a finite-free module of "fairly small rank." If $\mathscr{B}=\{(r, a)\}$ is the basket of $S$, then each $\left(1-t^{r}\right)$ appears in the denominator so that a first necessary condition for this is that each $r$ divides some $a_{i}$.

We have the obvious bound $\sum n \leq 19$ for the number and types of Du Val singularities $A_{n}, D_{n}$, and $E_{n}$ on a $K 3$ surface $S$. This implies the bound $\sum(r-$ $1) \leq 19$ for the basket $\mathscr{B}=\{(r, a)\}$ on a $K 3$ surface (see [6, Theorem III.9.20] and [7, Theorem II.8.21]). For a Fano 3 -fold $X$, a similar bound on the basket $\{(r, a,-1)\}$ is provided by an argument of Kawamata [8] who first proves that $A c_{2}>0$; then (3.2) implies

$$
\sum\left(r-\frac{1}{r}\right)<24
$$

Thus, there are only finitely many possibilities for the basket $\mathscr{B}$. An easy calculation shows that in either case, at most, 5 distinct values of $r$ occur.

In [2], we develop a procedure based on these ideas to find all possible Hilbert series for $K 3$ surfaces and Fano 3-folds with graded ring of given codimension. We give explicit lists [1] of codimension 3 and 4 cases (comparable to the lists of hypersurfaces and codimension 2 in Fletcher [7]) and, in most cases, settle the question of the existence of the varieties.

The numerical data of a $K 3$ surface $S$ with $D$ is $P_{1}=h^{1}(S, D)$, where $0 \leq P_{1} \leq$ $3+\operatorname{codim} S$ and the basket $\mathscr{B}=\{(r, a)\}$. We can rewrite $D^{2}$ from the formula of $P_{1}$ in terms of $P_{1}$ and the basket $\mathscr{B}$,

$$
D^{2}=2\left(P_{1}-2\right)+\sum \frac{a(r-a)}{r} .
$$

We produced the lists of codimension 3 and 4 [1] by searching all possible $K 3$ surfaces of given numerical data. We give an example below to show how this search is carried out. More details of applications are given in the Singapore paper [3].

EXAMPLE 4.1. A typical example is

$$
D^{2}=-2+\frac{1}{2}+\frac{1}{2}+\frac{3}{4}+\frac{4}{5}
$$

That is, the numerical data are the basket $\mathscr{B}=\{(2,1),(2,1),(4,1),(5,1)\}$ and $P_{1}=1$ (see formula (4.3)). This is \#39 in the codimension 3 list [1]. Since $P_{1}=1$, 
there is a generator of degree 1, say $x$. From formula (3.8),

$$
\begin{aligned}
P_{S}(t)= & \frac{1+t}{1-t}+\frac{t(1+t)}{2(1-t)^{3}} \frac{11}{20}-2 \times \frac{t}{2 \cdot 2\left(1-t^{2}\right)} \\
& -\frac{3 t+4 t^{2}+3 t^{3}}{2 \cdot 4\left(1-t^{4}\right)}-\frac{4 t+6 t^{2}+6 t^{3}+4 t^{4}}{2 \cdot 5\left(1-t^{5}\right)} .
\end{aligned}
$$

To kill its denominators, we must have at least two more generators, say $t_{1}$ and $v$, whose degrees are divisible by 4 and 5 , respectively. Therefore, the smallest choice of $\left(a_{1}, a_{2}, a_{3}\right)$ is $(1,4,5)$. After simplifying, we obtain

$$
(1-t)\left(1-t^{4}\right)\left(1-t^{5}\right) P_{S}(t)=t^{10}+t^{8}+t^{7}+2 t^{6}+t^{5}+2 t^{4}+t^{3}+t^{2}+1 .
$$

This looks like the Hilbert series of an Artinian ring with further generators $y$, $z, t_{2}$ of degrees $2,3,4$ so that the possible candidates $S$ is in the weighted projective space $\mathbb{P}(1,2,3,4,4,5)$ (see $[2,6,7]$ for definition). To find the structure of its resolution, multiply (3.6) again by $\left(1-t^{2}\right)\left(1-t^{3}\right)\left(1-t^{4}\right)$,

$$
\begin{aligned}
& (1-t)\left(1-t^{2}\right)\left(1-t^{3}\right)\left(1-t^{4}\right)^{2}\left(1-t^{5}\right) P_{S}(t) \\
& \quad=1-t^{6}-t^{7}-2 t^{8}-t^{9}+t^{10}+2 t^{11}+t^{12}+t^{13}-t^{19}
\end{aligned}
$$

From here, we read off the shape of the resolution of $R(S, D)$ over $A=k[x, y, z$, $\left.t_{1}, t_{2}, v\right]$, namely,

$$
\begin{gathered}
0 \longrightarrow A(-19) \stackrel{P^{t}}{\longrightarrow} A(-13) \oplus A(-12) \oplus A(-11) \oplus A(-11) \oplus A(-10) \\
\stackrel{M}{\longrightarrow} A(-9) \oplus A(-8) \oplus A(-8) \oplus A(-7) \oplus A(-6) \stackrel{P}{\rightarrow} A \longrightarrow R \rightarrow 0,
\end{gathered}
$$

where $P$ is a $5 \times 1$ vector and $M$ is a $5 \times 5$ skew-symmetric matrix. In other words, we expect 5 relations in degrees $6,7,8,8$, and 9 , and 5 syzygies in degrees $10,11,11,12$, and 13 . Note that $n=1+2+3+4+4+5$ corresponds to the canonical class of $\mathbb{P}(1,2,3,4,4,5)$ and hence the canonical divisor $K_{S}$ of $S$ is $O(19-n)$, which is trivial. The shape of the polynomial, together with the Buchsbaum-Eisenbud theorem on Gorenstein rings in codimension 3 (see [4]), gives us the equations of the relations as the Paffian of a $5 \times 5$ skew-symmetric matrix $M$ with degrees

$$
\left(\begin{array}{lllll}
0 & 2 & 2 & 3 & 4 \\
2 & 0 & 3 & 4 & 5 \\
2 & 3 & 0 & 4 & 5 \\
3 & 4 & 4 & 0 & 6 \\
4 & 5 & 5 & 6 & 0
\end{array}\right)
$$


Acknowledgments. This work derives mainly from the Ph.D. thesis [2], University of Warwick. I would like to thank both Miles Reid and Alessio Corti for their help and encouragement at different times.

\section{REFERENCES}

[1] S. Altınok, Lists of K3 surfaces in codimension 3 and 4, preprint, http:// www.maths.warwick.ac.uk/ miles/doctors/Selma.

[2] _ Graded rings corresponding to polarised K3 surfaces and $\mathbb{Q}$-Fano 3-folds, Ph.D. thesis, University of Warwick, Coventry, UK, 1998.

[3] S. Altınok, G. Brown, and M. Reid, Fano 3-folds, K3 surfaces, and graded rings, Singapore International Symposium in Topology and Geometry (NUS, 2001) (A. J. Berrick, M. C. Leung, and X. W. Xu, eds.), Contemp. Math., American Mathematical Society, Rhode Island, 2002, to appear.

[4] D. A. Buchsbaum and D. Eisenbud, Algebra structures for finite free resolutions, and some structure theorems for ideals of codimension 3, Amer. J. Math. 99 (1977), no. 3, 447-485.

[5] A. Corti, A. Pukhlikov, and M. Reid, Fano 3-fold hypersurfaces, Explicit Birational Geometry of 3-Folds (A. Corti and M. Reid, eds.), London Mathematical Society Lecture Note Series, vol. 281, Cambridge University Press, Cambridge, 2000, pp. 175-258.

[6] A. R. Iano-Fletcher, Plurigenera of 3-folds and weighted hypersurfaces, Ph.D. thesis, University of Warwick, Coventry, UK, 1988.

[7] __ Working with weighted complete intersections, Explicit Birational Geometry of 3-Folds (A. Corti and M. Reid, eds.), London Mathematical Society Lecture Note Series, vol. 281, Cambridge University Press, Cambridge, 2000, pp. 101-173.

[8] Y. Kawamata, Boundedness of Q-Fano threefolds, Proceedings of the International Conference on Algebra, Part 3 (Novosibirsk, 1989), Contemp. Math., vol. 131, American Mathematical Society, Rhode Island, 1992, pp. 439-445.

[9] M. Reid, Young person's guide to canonical singularities, Algebraic Geometry, Bowdoin, 1985 (Brunswick, Maine, 1985), Proc. Sympos. Pure Math., vol. 46, American Mathematical Society, Rhode Island, 1987, pp. 345-414.

Selma Altinok: Department of Mathematics, Arts and Science Faculty, Adnan Menderes University, Aydın 09010, Turkey

E-mail address: sa1tinok43@hotmai1.com 


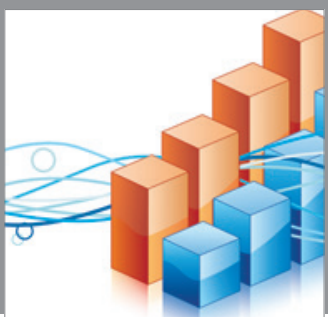

Advances in

Operations Research

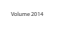

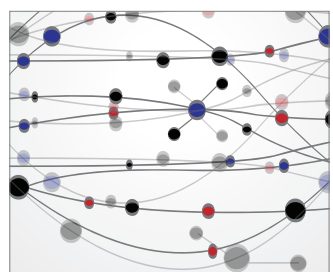

\section{The Scientific} World Journal
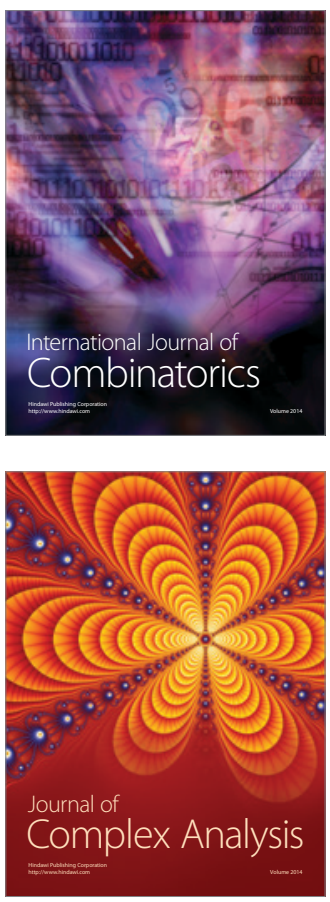

International Journal of

Mathematics and

Mathematical

Sciences
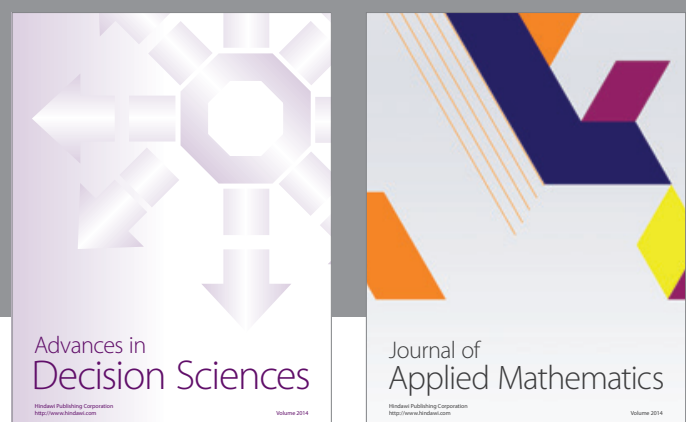

Journal of

Applied Mathematics
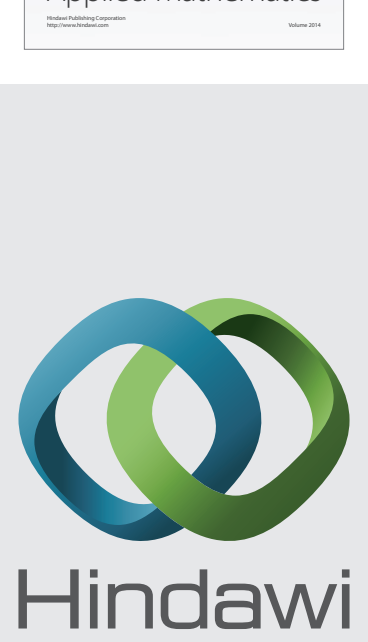

Submit your manuscripts at http://www.hindawi.com
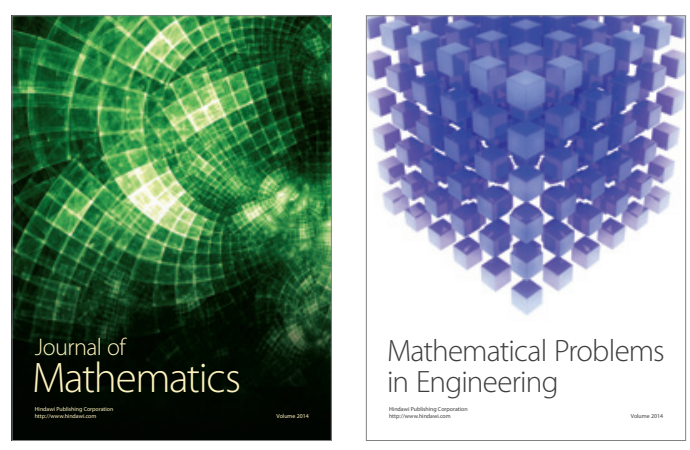

Mathematical Problems in Engineering
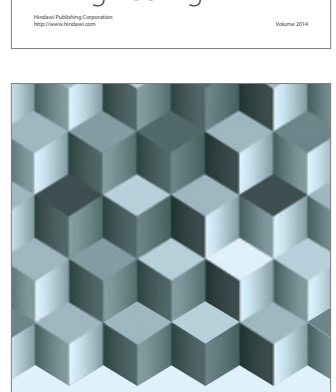

Journal of

Function Spaces
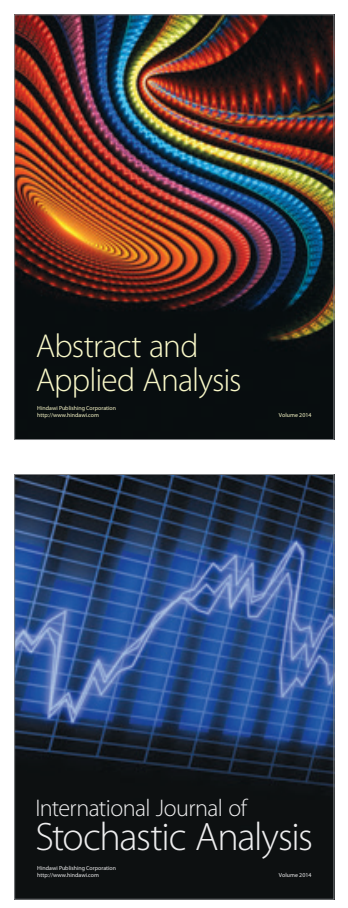

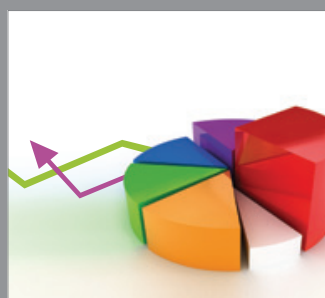

ournal of

Probability and Statistics

Promensencen
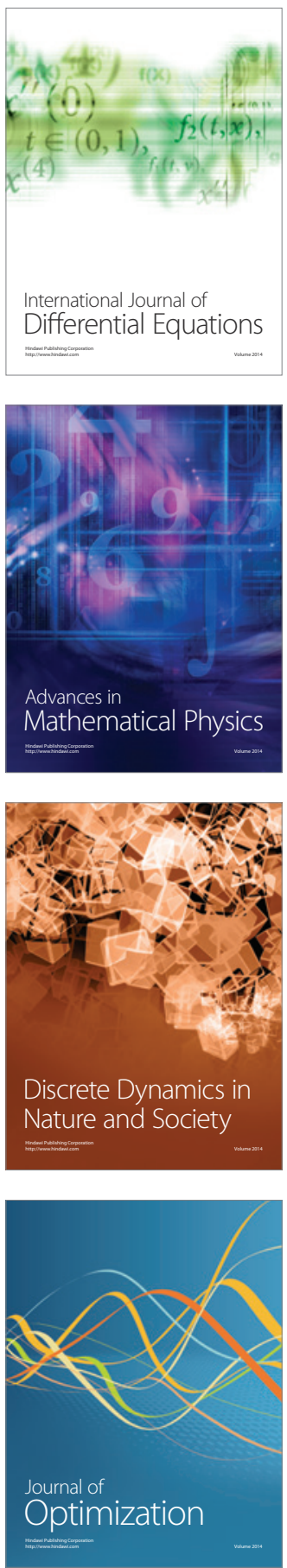